\title{
Limited evidence on risks of complications with invasive dental procedures in HIV-infected people
}

\section{Are people with HIV/AIDS at greater risk of complications from intra-oral dental procedures than HIV-negative people?}

Patton LL, Shugars DA, Bonito AJ. A systematic review of complication risks for HIV-positive patients undergoing invasive dental procedures. J Am Dent Assoc 2002; 133:195-203

Data sources English-language articles only, in Medline and EMbase from the early 1980s to April 2000.

Study selection Studies were selected that reported complications of a wide range of intraoral procedures if HIV status was confirmed by test results and outcomes or complications of infection (local or systemic) or delayed healing were included. Studies were excluded where treatment was not provided concurrently to HIV-positive and -negative individuals or outcomes were not reported.

Data extraction and synthesis Study design, number of patients, use of antibiotics and complication rate were recorded and a quality assessment of papers undertaken.

Results Only five studies could be included of which one examined endodontic treatment and the other four examined dental extractions. For dental extraction, unadjusted complication rates ranged from 2.9$13.9 \%$ in HIV-negative patients to $3.0-22.2 \%$ in HIV-positive patients. Conclusions The complications of invasive intra-oral procedures that were reported in HIV-positive patients were mild and easily treatable. Only limited evidence is available to guide clinicians, however.
Address for correspondence: Lauren Patton, Department of Dental Ecology, CB7450, School of Dentistry, University of North Carolina, Chapel Hill, NC 27599-7450, USA. E-mail: Lauren_Patton@dentistry.unc.edu

\section{Commentary}

With an ever-increasing number of people with HIV/AIDS, more dentists are likely to be treating HIV-infected patients. The question asked by Patton and colleagues is therefore very relevant to all general dental practitioners. In the early 1980s many HIV-positive people were seen in specially dedicated dental units but many of these have now been disbanded and patients with asymptomatic HIV infection are encouraged to seek dental treatment in general dental practices. This systematic review is very timely and answers a very practical question with only high-quality data. Although it may be that there are no other high-quality studies, it should be noted, however, that the review used only English-language literature and yet the highest incidence of HIV/AIDS is in nonEnglish-speaking countries.

Once again, one is struck by the low number of well-conducted trials that include controls and are of a prospective design. The conclusions that can be drawn are therefore very limited and only provide answers for two procedures: extractions and endodontics. In both these procedures, there is likely to be little difference in the rate of complications between HIV-positive or -negative patients.

Unfortunately, all these studies were conducted prior to more accurate estimates of status, such as viral load, and before the use of highly active antiretroviral therapy (HAART), and they did not divide patients according to disease progression. Any differences may be even smaller if the comparisons distinguished between asymptomatic HIV-infected people who have low viral loads and are prescribed HAART, symptomatic AIDS patients and HIVnegative individuals. It is also possible that new HIV therapies could cause complications such as increased root caries. There is therefore limited evidence that patients with HIV/AIDS who have no major medical problems can be treated in dental practice in the same way as all other patients.

HIV-infected people now survive longer and may increasingly often require orthodontic treatments and implants and highquality dental care. Multicentre cohort studies of HIV-positive populations undergoing all forms of dental treatment are necessary to discover whether there are any differences in complication rates or in the types of treatment required.

\section{Practice point}

- The available evidence suggests that HIV/AIDS patients can be treated in dental practice in the same way as other patients, but the quality and quantity of that evidence is limited.

\section{Joanna M Zakrzewska}

Oral Medicine, Barts and the London Queen Mary's School of Medicine and Dentistry, London, $U K$

Evidence-Based Dentistry (2003) 4, 15.

doi:10.1038/sj.ebd.6400159 